\title{
Management Technology: guidelines for innovation
}

\section{Tecnologia de Gestão: orientações para a inovação}

DOI: 10.46814/lajdv3n5-010

Recebimento dos originais: 01/07/2021

Aceitação para publicação: 31/08/2021

\author{
Wander de Moraes Paes \\ Mestrado (M.S) \\ Instituição de atuação atual \\ Endereço completo: Rua Senador Eusébio, 30, apto. 201. Flamengo - RJ \\ E-mail: wanderpaes@gmail.com
}

\begin{abstract}
Nowadays the concept and practice about technology management should be thought inside a complex environment in relation with the dynamics information came from other areas. A interdisciplinary concept involved with the dynamic relationship that should be oriented to harness collaboration and integration among the actors and knowledge into digital environment. This perspective reflects the technology management practices and concepts in the context related with other areas, therefore, this point of view is necessary to apply innovated process in order to develop product and service onto Internet environment. So, this article fills a knowledge gap lived currently in most organizations.

Objective: Development a set of directives to be considers during managements practices within organizations and project for developing product and services onto digital environment. This article does not have a proposal to present a method for enabling a kind of innovation solution, but a set of policies and recommendations necessary to achieve the best result in order to develop a product and services via innovation mindset.

Method: Review and analysis problems with adaptive comprehension involved with management practices and concept. We present these approaches with and interrelationship environment, which can improve the comprehension analyzing approaches from others areas and body of knowledge. This mindset is necessary to mitigate many of the problems and improve the innovation idea applied on developing product and services on internet environment. We present this concept of a "management oriented to innovation" as a pattern of thinking to be argue from managers, used to perform an innovation process and task at various levels and development product and services. A kind of prototype implementation called Innovation that is a process related with a some others areas outside technology .

Results : On the basis of these analyses, we propose a policy and guidelines for supporting an innovation initiative is a result of interactions with others areas and concept not necessary inside of technology respects. We develop an mind set architecture for innovation system based on the metaphor of a "innovation". Finally, we describe this architecture, with an interface with others concept, for planning and implementing products and services for WEB. A set of adaptive and interrelationship subjects to insight management practices necessary to improve and follow the tendency of technology applied to new market and business.

Conclusion: This article offers multi-level reflections and references to enhance the performance manager skill at manageable practices to deal with developing process to create innovative environment, products and services for digital environment.
\end{abstract}

Keywords: Technology Management, Complexity, Systems Architecture, Knowledge Management, Strategy Planning, Innovation. 


\section{RESUMO}

Hoje em dia, o conceito e a prática sobre gestão tecnológica devem ser pensados dentro de um ambiente complexo em relação com a informação dinâmica proveniente de outras áreas. Um conceito interdisciplinar envolvido com a relação dinâmica que deve ser orientada para aproveitar a colaboração e a integração entre os actores e o conhecimento no ambiente digital. Esta perspectiva reflecte as práticas e conceitos de gestão tecnológica no contexto relacionado com outras áreas, portanto, este ponto de vista é necessário para aplicar processos inovadores a fim de desenvolver produtos e serviços no ambiente da Internet. Assim, este artigo preenche uma lacuna de conhecimento vivida actualmente na maioria das organizações.

Objectivo: Desenvolver um conjunto de directivas a serem consideradas durante as práticas de gestão dentro das organizações e projectos de desenvolvimento de produtos e serviços para o ambiente digital. Este artigo não tem uma proposta para apresentar um método que permita uma espécie de solução inovadora, mas um conjunto de políticas e recomendações necessárias para alcançar o melhor resultado, a fim de desenvolver um produto e serviços através de uma mentalidade inovadora.

Método: Revisão e análise de problemas de compreensão adaptativa envolvidos com práticas e conceitos de gestão. Apresentamos estas abordagens com e ambiente de inter-relação, que podem melhorar a compreensão analisando abordagens de outras áreas e corpo de conhecimento. Esta mentalidade é necessária para mitigar muitos dos problemas e melhorar a ideia de inovação aplicada no desenvolvimento de produtos e serviços no ambiente da Internet. Apresentamos este conceito de "gestão orientada para a inovação" como um padrão de pensamento a ser defendido pelos gestores, utilizado para realizar um processo e tarefa de inovação a vários níveis e desenvolvimento de produtos e serviços. Uma espécie de implementação de protótipo chamada Inovação que é um processo relacionado com algumas outras áreas fora da tecnologia .

Resultados : Com base nestas análises, propomos uma política e orientações para apoiar uma iniciativa de inovação é o resultado de interacções com outras áreas e conceitos não necessários dentro dos aspectos tecnológicos. Desenvolvemos uma arquitectura de conjunto de mentes para um sistema de inovação baseado na metáfora de uma "inovação" . Finalmente, descrevemos esta arquitectura, com uma interface com outros conceitos , para o planeamento e implementação de produtos e serviços para a WEB. Um conjunto de temas adaptativos e de inter-relação com as práticas de gestão de insight necessárias para melhorar e seguir a tendência da tecnologia aplicada a novos mercados e negócios.

Conclusão: Este artigo oferece reflexões e referências a vários níveis para melhorar a capacidade do gestor de desempenho em práticas geríveis para lidar com o desenvolvimento de processos para criar um ambiente inovador, produtos e serviços para o ambiente digital.

Palavras-chave: Gestão Tecnológica, Complexidade, Arquitectura de Sistemas, Gestão do Conhecimento, Planeamento Estratégico, Inovação.

\section{INTRODUÇÃO}

O desenvolvimento desse artigo apresenta o conceito e práticas sobre Gestão Tecnológica relacionada ao ambiente complexo no que tange à dinâmica do ambiente informacional contemporâneo. Uma abordagem sobre um ambiente informacional relacionado ao contexto sóciotecnológico com mudanças frequentes numa dinâmica que envolve a colaboração e integração entre os atores no meio digital. O que traduz numa perspectiva da Gestão de Tecnologia representada pela interoperabilidade das ações em diversas áreas de conhecimento relacionadas aos processos e conceitos necessários para inovação tecnológica aplicada ao mercado do meio digital. 
A consolidação da tecnologia Internet como plataforma de inovação e desenvolvimento de soluções, sob a perspectiva da colaboração das informações, influenciou diretamente as soluções de negócio e os sistemas computacionais. Essa plataforma tecnológica dos serviços descentralizados representa um impacto significativo no processo de inovação de produtos e serviços no ciberespaço no que tange aos aspectos sobre arquitetura de sistemas, integração, colaboração, gestão, e interação homem-máquina.

Portanto, o objetivo específico desse artigo é apresentar a ideia do ambiente complexo da Gestão de produtos e serviços no ambiente sob a tecnologia Internet como necessidade nos modelos de gestão que relaciona aspectos tecnológicos das redes sem fio, arquitetura de sistemas, liderança, planejamento estratégico, e gestão de conhecimento. Um cenário que versa necessita de diretrizes interrelacionadas com outras áreas de conhecimento para lidar com as novas abordagens da Gestão orientada à inovação tecnológica que abrange os aspectos Administrativos, Organizacionais, Tecnologia de sistemas, Gestão de conhecimento e Mercado/Negócios. Essa abordagem representa a reflexão da influência desses aspectos para construção de um ambiente inovador necessários para uma organização que possui como objetivo principal o desenvolvimento de produtos e serviços no meio digital. E, sobretudo, enquadra também a ideia da Gestão Tecnológica de uma maneira multidisciplinar e Interdisciplinar, e consequentemente revela a administração do meio digital como parte integrante do processo de inovação tecnológica para a colaboração das informações e integração no ciberespaço. Um ambiente que apresenta atualmente um "Background" na qual os gestores, em boa parte das organizações, não possuem percepção e a capacitação de atuar num ambiente complexo de mudanças constantes e com diversas influencias oriundas das demais áreas de conhecimento. Informações para agregar um perfil adaptativo e orgânico necessários ao gestor contemporâneo a fim de criar a consciência para trabalhar num sistema dinâmico e com tecnologias disruptivas que influenciam diretamente a tendência e a sobrevivência das soluções sistêmicas no mercado digital. Uma ideia carregada de conceitos relacionados e multidisciplinares com diretrizes de atuação para nortear a implementação de um ambiente inovador ao desenvolvimento de produtos e serviços competitivos no mercado.

\section{COMPLEXIDADE}

O ambiente informacional contemporâneo apresenta um contexto socio-tecnológico dinâmico com mudanças constantes e intensas, representando novas abordagens para compreensão e administração das iniciativas no meio digital, bem como para percepção da integração e colaboração das informações. Essa abordagem carrega uma nova perspectiva sob a ótica da Complexidade com conceitos mais aderentes para interpretação dos fenômenos distribuídos no ciberespaço que não está 
mais restrita somente ao desenvolvimento de sistemas/aplicativos, mas também aos aspectos sociais, políticos e administrativos.

Em relação aos aspectos das praticas de gestão, diversos fatores influenciam a estratégia dos projetos e iniciativas para desenvolvimentos de soluções inovadoras meio digital. Os fenômenos da Complexidade auxiliam nessa nova perspectiva de perceber e pensar o meio, dentre as quais podemos ressaltar:

i. Sistemas Dinâmicos : ambiente multivalorado com relacionamentos diretos e indiretos. Onde a dinâmica do meio proporciona o equilíbrio e a caracterização de um contexto.

ii. Atratores : atores que agregam relações diretas e indiretas e criam relações de poder para influenciar o meio .

iii. Auto Organização : característica de se auto-organizar de acordo com as relações do meio.

iv. Força : ideia que a força não é causal. Sempre se apresenta relacionada ao meio e aos atores, direta e indiretamente .

v. Emergência : surgimento de uma tendência ou fenômeno oriundo do dinamismo das relações .

vi. Campo : o conceito de campo de atuação como espaço que determina as relações e os elementos do meio.

vii. Estratégia : num espaço complexo é fundamental trabalhar com estratégia, criando relações colaborativas que garantirão a sobrevivência no meio .

Essa abordagem permite nova abstração para interpretação de um ambiente informacional com a proposta de fluidez que permite uma visão mais aderente com a dinâmica dos processos e fluxo das informações relacionadas à tecnologia. Num contexto que molda o conteúdo, apresentando o texto do contexto, evidenciando a limitação dos recursos e ideias, e emergência de novos cenários, com objetivo de transformar essas limitações em recursos para criação de novas abordagens e interpretações. Esse aparente paradoxo promove o desenvolvimento fora das interpretações monolíticas, estáticas e estagnadas de como se interpretar um ambiente informacional que contribui para as práticas de gestão tecnológica para o desenvolvimento de produtos e serviços no ambiente dinâmico da Internet. Quando percebemos alguns exemplos da interpretação dos fenômenos sobre Complexidade aplicada numa abordagem inter-relacionada nas áreas de Tecnologia(i), Administração/Gestão/Governança (ii), e Mercado Financeiro (iii), conforme descritos abaixo: 
i. Na área de Tecnologia podemos utilizar o conceito das Redes e Atratores para representar a distribuição e a dinâmica da topologia das Redes Sociais no meio digital. Bem como a disposição das suas relações e influência.

ii. Na área Administração (Gestão/Governança), a emergência das novas soluções e alternativas está diretamente relacionada ao planejamento estratégico para o desenvolvimento de produtos e serviços. Para exemplificar, imagine um planejamento estratégico que proporciona um investimento em diversos segmentos. Daí, desse ambiente, emerge nova(s) alternativa(s) das relações no meio por meio de novos investimentos oriunda das relações/soluções pré-existentes. Uma combinação de alternativas existentes que fomenta e contribui a criação de outro produto.

iii. No Mercado financeiro, soluções sistêmicas que relacionam conceitos da Física como Força, Campo e Volume em conjunto com práticas da área de Economia com objetivo de valorar e prever variações das ações das empresas negociadas na Bolsa de Valores distribuídas no mercado. Conceito intitulado de Econofísica 5 .

Importante ressaltar também que um ambiente informacional é composto por pessoas (Atores), com dinamismo e mudanças, inerentes ao comportamento humano. Dessa forma, a gestão do relacionamento e da colaboração no ambiente é conveniente para afrouxar os vínculos e fomentar os pensamentos fora do padrão, com objetivo de uma convivência harmônica e sistêmica entre o formal e o informal. Nessa abordagem, a gestão desse conhecimento relaciona não somente o que se aprende, mas a maneira como se aprende, se percebe, e modifica o meio que permeia por toda rede de informação de um ambiente informacional. O que traduz numa necessidade da visão multidisciplinar das praticas de Gestão num ambiente dinâmico de mudanças constantes para se adaptar à sobrevivência de mercado que envolve toda cadeia de valor que, por conseguinte, engloba o processo do desenvolvimento de produtos e serviços. Um novo discurso em sintonia com as novas necessidades para inovação em resposta ao pensamento tradicional linear.

\begin{tabular}{|l|l|}
\hline Discurso do pensamento linear e sistêmico. & Discurso do pensamento Complexo. \\
\hline Não perca o foco. & $\begin{array}{l}\text { Alterne as visões focada (redutivistas) e periférica (ampliadora) segundo } \\
\text { os contextos,culturas, e as necessidades. }\end{array}$ \\
\hline Use sempre as melhores práticas. & Mude as práticas segundo os momentos, as circunstâncias e os contextos. \\
\hline A realidade é repetitiva. & A realidade é mutante e inter-relacionada. \\
\hline $\begin{array}{l}\text { É preciso eliminar totalmente o erro, a } \\
\text { incerteza e a ilusão. }\end{array}$ & É preciso diminuir o erro, a incerteza e a ilusão. \\
\hline $\begin{array}{l}\text { As pessoas que estão nos cargos também } \\
\text { estão no controle. }\end{array}$ & As pessoas que estão nos cargos nem sempre estão no controle. \\
\hline
\end{tabular}




\section{GOVERNANÇA PARA INOVAÇÃO}

A ideia que envolve o conceito da Inovação está diretamente relacionada à colaboração inserida num ambiente multidisciplinar e inter-relacionado com a proposta de articular e criar processos para resolverem os objetivos de negócio dos novos produtos e serviços. Processos que introduzem na cadeia de valor de uma organização um novo produto, serviço, método, ou material para aplicação comercial ou qualquer objetivo prático específico. Dessa forma, as principais diretrizes propostas nesse artigo, no que tange ao desenvolvimento de produtos e serviços, estão segmentadas em 7 abrangentes abordagens de iniciativa que devem ser consideradas no desenvolvimento de soluções inovadoras no mercado. De modo que as praticas de gestão de tecnologia precisam considerar com relevância essas 7 diretrizes com objetivo de aplicar e administrar o processo de inovação dos produtos e serviços no meio digital:

\subsection{PLANEJAMENTO ESTRATÉGICO}

Importante elucidar a abordagem sobre o Planejamento e Estratégia como forças coexistentes na abordagem que chamamos de Planejamento Estratégico, mas que pertencem a campos de atuação distintos na sua concepção. A estratégia está relacionada à ação, como articulação dessas ações em prol de um objetivo. E o planejamento com a ideia de formatação pela qual detalhamos e controlamos as ações na linha do tempo (como num cronograma, por exemplo). Uma dinâmica que cria um conceito que “é estratégico planejar" em conjunto com a ideia que também "planejar é estratégico", representando uma abordagem importante para situar a necessidade de aplicação desses conceitos no processo de inovação. De modo que no planejamento onde se formata as metas a serem alcançadas sujeita às mudanças, controle e influência das estratégias. Uma ideia de organizar e criar um pensamento de atuação orientado para atingir um objetivo(os) no futuro. O que traduz numa ação articulada de planejamento e estratégia carregada com as necessidades de mudanças inerentes aos sistemas dinâmicos, mutantes, político, com necessidades efêmeras e necessárias ao mercado contemporâneo. Mas que necessitam estar mapeadas nesse relativo controle do planejamento estratégico.

\subsection{ESTRUTURA ORGANIZACIONAL}

A Estrutura Organizacional, carregadas dos seus processos e responsabilidades internas, sustenta o pilar que proporciona o ambiente do desenvolvimento das atividades colaborativas para inovação tecnológica. Isso representa a formatação de como uma organização se caracteriza e interrelaciona seus departamentos internos para atuar em prol dos objetivos, e projetos dos produtos e serviços no meio digital. O foco nesse capitulo não é abordar todas as alternativas ou a melhor estrutura 
organizacional de uma organização, pois isso representa um assunto bastante extenso. A ideia é apresentar a necessidade de uma estrutura para receber os movimentos de interação interna e externa ao ambiente , em conjunto com a consideração da importância de uma estrutura administrativa entre as áreas/equipes que autua e influenciam uma organização. Pois, essa estrutura sócio-politicoadministrativa é quem permite a criação de novos relacionamentos para emergência de novos projetos que estimulam a inovação de produtos e serviços de maneira mais orgânica e competitiva para o retorno de valor de uma instituição. Que podemos salientar algumas atuações que representam a habilidade de um gestor em lidar com estratégias administrativas que proporcionem uma melhor política organizacional, adaptada a sua cultura, que dentre outros aspectos, orientam a atuação dos representantes da empresa no processo inovador de produtos e serviços no meio digital :

Centralizado : A ideia de centralizar uma estrutura que proporciona o desenvolvimento das ideias e projetos de novos produtos e serviços se caracteriza pelo ambiente concentrado nas dependências internas da empresa sob composição e administração exclusiva da equipe de funcionários internos da empresa, relacionados numa área/setor que tem por objetivo principal e especifico o fomento dessa atuação, como numa área de $\mathrm{P} \& \mathrm{D}$ (desenvolvimentos de produtos).

Externo : Como no centralizado, mas num ambiente externo, fora das dependências da Matriz que se formatam como Parcerias/Consultorias por meio de contratos com outras empresas (parceiras de negócio) para apoiar ou compor a criação da equipe e processos responsáveis pela inovação dos produtos e serviços da empresa. Bem como as parcerias de negócio com empresas para troca e/ou aquisição de novas tecnologias e processos (Joint Ventures, Licenças de uso, Alianças estratégicas, etc.) com objetivo de incorporar essas novas tecnologias e processos na cadeia de valor já existente na organização.

Rede : Criar redes de relacionamento de colaboração (Internas e externas a empresa) e troca de experiências, informações e ideias para desenvolvimento de projetos e fomento de ideias para novos produtos e serviços .

StartUp : estratégia de investimento em produtos de propostas e iniciativas de terceiros que precisam de capital de investimento. Uma vez que o sucesso do produto alcançar índices satisfatórios de consumo no mercado, é incorporado na cadeia de valor dos produtos e serviços já existentes na organização. 
Processos Àgeis: Importante ressaltar a utilização dos processos de desenvolvimento orientados à colaboração, desburocratizados, mais adaptativos sob os conceitos de administração Ágil ${ }^{1}$ para acompanhamento e gerenciamento dos projetos de desenvolvimento de produtos e serviços.

\subsection{FINANÇAS/INVESTIMENTO}

A ideia sobre o controle/criação de orçamento para investimentos nas iniciativas inovadoras é apresentar uma estratégia de controle e seleção dos recursos para aplicação em projetos de acordo com as recomendações da BBRT (www.bbrt.org) de gerenciamento flexível dos orçamentos orientados aos processos de Inovação e desempenho financeiro dos produtos e serviços. Na qual a ideia tradicional de $\mathrm{CAPEX}^{2}$ e $\mathrm{OPEX}^{3}$ que direciona os investimentos na organização com objetivo de adquirir um perfil flexível mais aderente ao contexto dinâmico das necessidades do mercado que deve refletir no processo das iniciativas de desenvolvimento dos produtos e serviços focado na manutenção financeira da cadeia de valor desses produtos e serviços e no retorno de investimento . O que necessita de um controle de recursos mais flexível e suscetível ao risco e às mudanças constantes das necessidades do negócio. De modo que o orçamento inicial reservado aos projetos podem, e geralmente sofrem alterações para contemplar novas funcionalidades ou novas necessidades para se adaptar as estratégias comercias no decorrer do projeto. Sobretudo, não há intenção de recomendar a ausência de um planejamento financeiro nos projetos estratégicos e de risco numa organização, mas uma abordagem adaptativa com um plano de contas focadas nas cadeias de valores com respectivos centros de custos autônomos na qual o gestor poderá decidir pela própria aquisição, mudança ou remanejamento de recursos específicos inerentes a cada iniciativa ou projeto. A possibilidade de remanejamento dos recursos de maneira mais dinâmica entre os centros de custo seria para contemplar sub-projetos, mudanças de estratégias, ou atender à outros projetos relacionados tecnologicamente ou comercialmente mais aderentes aos objetivo ou missão da empresa no momento, como também remanejar recursos que foram otimizados no decorrer dos projetos. Sem deixar de observar que a otimização de recursos sempre deve ser o mote em qualquer empreendimento. Um controle que deve envolver o crivo do gestor responsável e até mesmo os responsáveis das áreas correlatas; como num comitê autônomo com finalidade especifica de direcionar os recursos no escopo dessas iniciativas. Um processo que se encontra fora da área de

\footnotetext{
${ }^{1}$ Processos de desenvolvimento ageis de produtos : KANBAN, SCRUM, XP, DDD - Domain Driven Design, Lean, Crystal, Open UP, etc

${ }^{2}$ CAPEX é o acrônimo de Capital Expenditure voltado para despesas de investimento em bens de capital que direciona os investimentos para aquisição (melhorias) de bens de capital .

${ }^{3}$ OPEX é o acrônimo de Operational Expenditure que relaciona o investimento associado à manutenção dos equipamentos e outras despesas operacionais, necessários à produção e à manutenção em funcionamento do negócio ou sistema.
} 
aquisição já existente e consolidada numa organização, a fim de adquirir flexibilidade e velocidade nas aquisições necessárias.

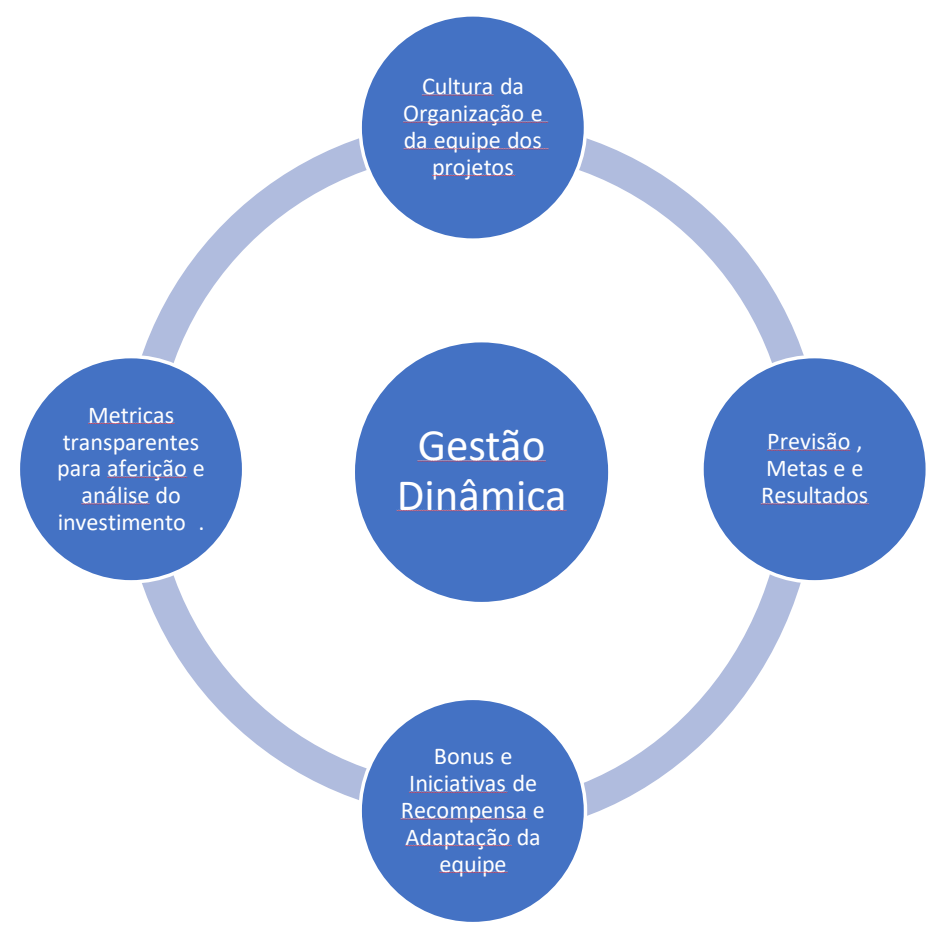

\subsection{MERCADO}

A análise de mercado está relacionada às estratégias de apresentação e introdução de um produto e serviço no mercado consumidor. Uma decisão que melhor atende às expectativas de retorno de investimento e aos critérios de utilização e consumo. O que envolve a estratégia de mercado em conjunto com a propaganda (marketing) do produto ou serviço.

Podemos salientar inicialmente 3 tipos de estratégia: o de ser o primeiro (pioneiro e inédito) no lançamento de um produto ou serviço no mercado; ou em conjunto ( ao mesmo tempo) com outros produtos já existentes; ou posterior quando demais produtos já se apresentaram consolidados no mercado. Cada qual com suas vantagens e desvantagens no que tange ao retorno de investimento e retorno de valor nos processos de inovação da organização. Sem deixar de considerar as práticas de propaganda (Marketing) nos canais de distribuição e apresentação das mídias (Jornais, TV, email, Radio, Pontos de Venda, Outdoor, Mídia impressa especializada, Internet, etc.). Um conjunto de estratégias de mercado que devem ser consideradas para introdução no mercado. Dentro dessa estratégia, é importante salientar uma atuação que está se tornando praxe na introdução no mercado consumidor, que é a de fornecer funcionalidades gratuitas , com objetivo de fidelizar a utilização pelo consumidor e também de criar possibilidades de um "cross-selling" (venda por transitividade) por meio das demais funcionalidades de um produto ou serviço. 


\subsection{GESTÃO E TECNOLOGIA DE SOFTWARE}

A área de tecnologia é abrangente com diversas áreas de atuação como Infraestrutura de Servidores, Redes, Segurança, Conectividade, Telecomunicações, e Software Básico (Sistemas Operacionais e protocolos). Mas a questão da tecnologia numa visão exclusivamente técnica, não é suficiente para superar os desafios exigidos pelo mercado. A busca da arquitetura tecnológica adequada está diretamente relacionada ao modelo dea gestão no desenvolvimento de produtos que relaciona os conceitos sobre Arquitetura de Sistemas e toda cadeia de valor de pessoas numa organização a fim de promover a interface de integração e aos anseios comerciais dos produtos e serviços. Essa abordagem promove a topologia e arquitetura de software como um ambiente a ser considerando na construção das soluções sistêmicas traduzidos nos produtos e serviços sob a ótica de uma gestão inovadora e sustentável como já se apresenta nas praticas, métodos e base de conhecimento como SAFe (Sacaled Agile Framework - www.scaledagile.com), SCRUM - www.scrum.org, XP http://www.extremeprogramming.org/, dentre outros.

Dessa forma, as arquiteturas são inseridas no modelo de gestão e se tornam mais focadas para inovação e necessidades comercial de mercado. Na qual podemos ressaltar as arquiteturas de sistemas (frameworks) direcionados para construção e desenvolvimento/construção de produtos como J2EE, J2SE, Aqualógic BEA, NET, Windows CE, J2ME, dentre outros. Também podemos ressaltar arquiteturas funcionais que agregam requisitos de negócio mais específicos como os CMS (Joomla, Drupal, OpenCMS, etc.), arquitetura para marketplace como o Catarse, e para redes sociais como o JomSocial, Bodypress, Anahita, para E-commerce como o Magento e OS Commerce, e para Workflow como o Alfresco, dentre as demais iniciativas "open source" que proporcionam ambientes com funcionalidades de negócios adequadas ao consumo/utilização sem custo de aquisição, proporcionando uma plataforma inicial agregada aos componentes de novas funcionalidades de acordo com a necessidade do negócio. O que traduz numa iniciativa que já proporciona um valor agregado aderente ao acréscimo de funcionalidades necessárias ao retorno de investimento funcional e/ou financeiro do produto ou serviço em questão.

Dessa forma, podemos considerar o conceito sobre arquitetura de sistemas como a plataforma de desenvolvimento de software sob 2 macro aspectos: um relaciona o que chamamos de Estilos Arquiteturais na qual os "frameworks" de desenvolvimento estruturam o ambiente onde o software (produto) será executado .

Exemplos de estilos arquiteturais :

i. WEB : composto de uma camada de apresentação (navegador), Programacao em Nuvem, WebServer, Applicattion Server, e uma camada de Banco de Dados. 
ii. Cliente Servidor : composto por um cliente e um servidor de aplicação configurado conectados remotamente por meio de uma rede.

iii. Dutos e Filtros : caracterizado pela disposição de módulos em sequencia no qual as rotinas especificas de transição são requisitadas por meio de passagem ou não de parâmetros sem dependência e coesão entre os módulos. Onde cada módulo devolve, fazendo a mediação/filtro e devolvendo novos parâmetros/informações para executar outros módulos com funcionalidades especificas. (Por ex: Processos Script-Batch, Listener).

iv. Orientada a Objetos : desenvolvido por meio de abstração de dados onde os componentes (componentes) representam os objetos do negócio mapeados no processo de requisito/análise do sistema. Onde o objetivo é abstrair os modelos de negócio, preservando as características numa eventual mudança, sem impactar os demais objetos (componentes) que seguem as principais características de Polimorfismo, Herança, Classe, Métodos, Mensagem, e Encapsulamento.

v. Orientada a Eventos : tradicionalmente, um sistema construído com uma interface que aciona e orquestra um conjunto de eventos ("Functions/Procedures") remotas e/ou locais com objetivos específicos do negócio .

Conforme descrito no segundo parágrafo dessa seção, os exemplos de "frameworks" utilizados nos estilos arquiteturais estão também divididos em 2 categorias: um relacionado para construção, e o outro caracterizado como plataformas agregado com funcionalidades mais especificas de negócios.

Nesse modelo orientado à arquitetura, o foco da solução sistêmica está relacionado ao mapeamento e utilização inicial dos valores já existentes na plataforma, com objetivo de agregar demais funcionalidades e auxiliar na organizar o código para futuras manutenções por meio da componentização. Componentes representados especificamente pelos serviços de software responsáveis pelas funcionalidades específicas agregadas na estrutura de software (arquitetura) do produto, que são submetidas a mudanças constantes, permitindo maior aderência às mudanças e inclusão de novos componentes (funcionalidades) para acompanhar de maneira eficaz as mudanças exigidas pelo mercado.

\subsection{CULTURA}

A gestão do conhecimento (GC), orientado à inovação tecnológica proposta nesse artigo, apresenta 2 macro-pontos a serem observados para aplicação dessa área de conhecimento num ambiente informacional responsável pela tecnologia da informação. A primeira é sobre o conceito da inovação tecnológica como pilares de referencia a serem aferidas e consideradas na relação Informação x Conhecimento, compreendidas e aplicadas numa organização para alcançar seus objetivos 
estratégicos. A outra é a percepção do ambiente para compreensão/percepção da atuação dos integrantes das equipes para adequá-los de acordo com direcionadores

estratégicos dos gestores da organização (visão, missão, objetivos estratégicos, projetos, gestão, e metas). A forma conceitual fundamenta, em linhas gerais, as 4 políticas do conhecimento de um ambiente:

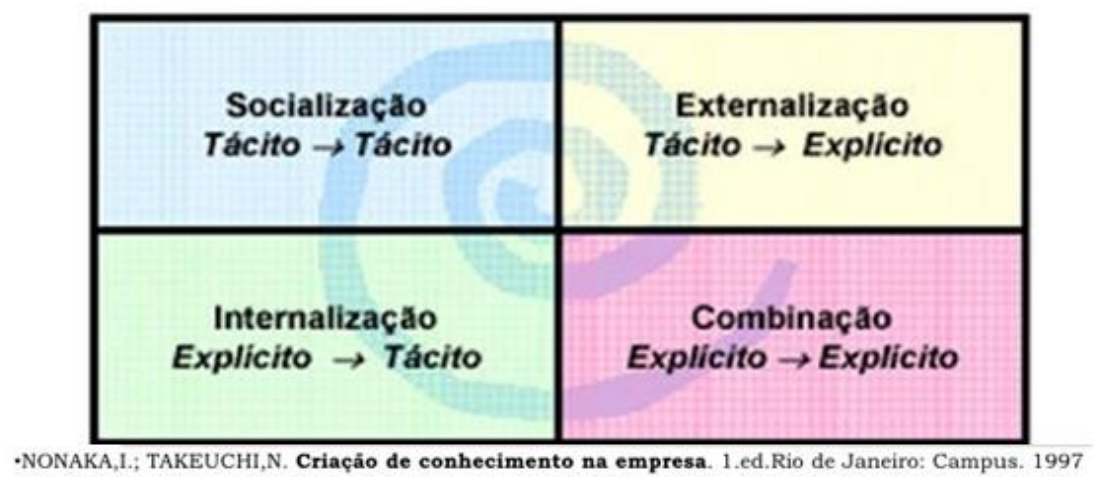

Socialização : Conhecimento compartilhado (Brainstorming). Se caracterizam por politicas coletivas e individuais de compartilhamento das informações dos projetos no que tange a tecnologia, regras de negócio, e objetivo da empresa .

Externalização (Socialização e colaboração) - http://www.innocentive.com/ - crowdsourcing de ideias. Combinação : Conhecimento Operacional construído pela cadeia de valor existente dos produtos e serviços. Relacionar de maneira virtuosa os envolvidos na cadeia de valor já existente nos processos de inovação e novos projetos. A identificação do conhecimento tácito dos integrantes das equipes traduzido pelo talento no desenvolvimento e ideias sobre os produtos comercializados na organização. Em conjunto com o conhecimento formal (explicito) que pode ser traduzido e divulgado por meio das informações aos integrantes das equipes.

Internalização: Conhecimento (Aprender fazendo - Go See/Gemba ${ }^{4}$ - Política de capacitação interna da organização em novas tecnologias e tendência. Utilizadas ou não pela empresa..

Considerando em cada política acima, os componentes que regem a dinâmica desse contexto como o Discernimento e respeito das idéias, Experiência (Tacita e Explicita), Verdade Fundamental (Consenso), Complexidade (Ambiente Dinâmico e Multivalorado), Verdade Prática (reflexão dos resultados práticos), Valores e Crenças (Ética).

\footnotetext{
${ }^{4}$ Sair da mesa ou escritório e agir também no local de trabalho onde o valor é produzido e os produtos são criados e usados.
} 


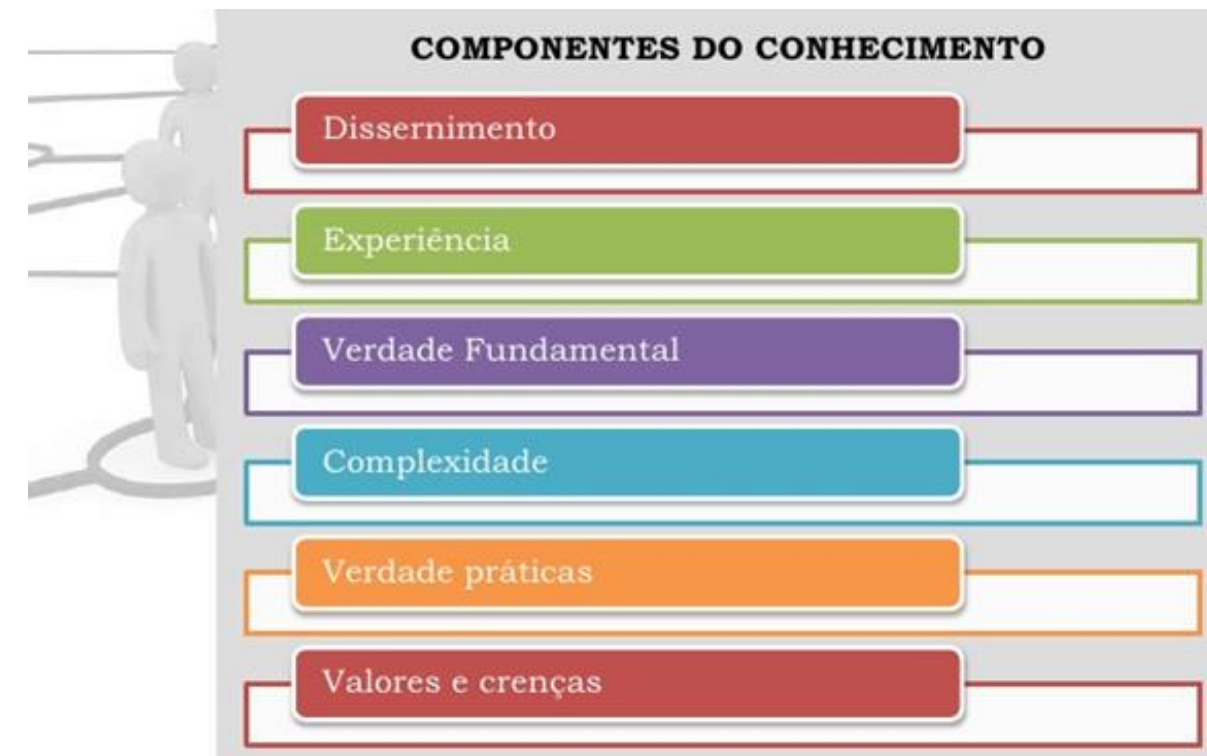

•NONAKA,1.; TAKEUCHI,N. Criaçáo de conhecimento na empresa. 1.ed.Rio de Janeiro: Campus. 1997

Entretanto, soluções para análise do "clima" de um ambiente e enquadramento dos "perfis" de atuação das pessoas num contexto de maior desempenho (baseado nas suas qualidades), apresentam uma ferramenta importante que podemos destacar 2 soluções com o intuito de elucidar a existência e recomendação de análise, dentre outras existentes no mercado. Uma é o método $O K A$ (Organizational Knowledge Assessment) e um outro exemplo é o PAT® Perfil . Uma vez dentro das regras de aferição dos seus métodos, apresentam um diagnóstico da cultura do ambiente para análise e tomada de decisão sobre as equipes que compõe um ambiente informacional.

\subsection{GESTÃO}

Os conceitos sobre Gestão inter-relacionam as demais diretrizes supracitadas, caracterizando um perfil de atuação multidisciplinar/interdisciplinar do gestor. Não acredito que e exista uma definição universal sobre Gestão. De modo que nesse artigo, a ideia é apresentar mais um ponto de vista sob os assuntos que envolvem as práticas necessárias para atuação de um Gestor. Que de maneira geral relaciona, desenvolve, e decide sobre a dinâmica de um ambiente informacional com objetivo de agregar valor e garantir a sobrevivência no meio em que a organização atua, utilizando os conceitos das 6 demais diretrizes supracitadas nos pontos desse capitulo.

O processo para uma gestão inovadora carrega conceitos das demais diretrizes com objetivo de perceber uma visão sistêmica e integrada. Dessa forma, a gestão da tecnologia está inserida num cenário inter-relacionado de ações e informações, num contexto sócio- tecnológico uma vez que o "ser humano" é quem desenvolve e utiliza a tecnologia e transforma cenários como agente inovador. 
A prática da Gestão se apresenta como mais uma variável que exerce influência nas demais variáveis de um ambiente informacional. Uma prática aplicada a um ambiente representado pelo esforço administrativo em busca do equilíbrio dos fenômenos do meio, sempre com objetivo de retorno do investimento (funcional, social ou financeiro) para sobrevivência de um produto ou serviço num mercado de atuação da organização. Um contexto de ações pessoais que está diretamente ligado à compreensão e ação (estratégia) num cenário complexo onde os movimentos internos e externos permeiam e influenciam de maneira direta e indireta toda cadeia da Sociedade da Informação.

Entende-se como Sociedade da Informação ${ }^{3}$ a dinâmica das atividades sociais, tecnológicas, econômicas e políticas que influência toda cadeia de um ambiente informacional. Uma cadeia que relaciona as atividades de inovação das soluções sistêmicas e utilização das informações no meio digital, na qual podemos ressaltar: (i) o processo do desenvolvimento das arquiteturas sistêmicotecnológicas regidas pelos próprios modelos e práticas, (ii) das relações de poder que ora articulam e ora desarticulam o planejamento das inovações tecnológicas, (iii) políticas públicas, (iv) e demais aspectos financeiros e mercadológicos como prazo, mudanças constantes, estratégias de mercado, governança tecnológica, etc. Que em conjunto representa atuações e conceitos que devem ser consideradas na prática da gestão para questões relacionadas à inovação das soluções no meio digital.

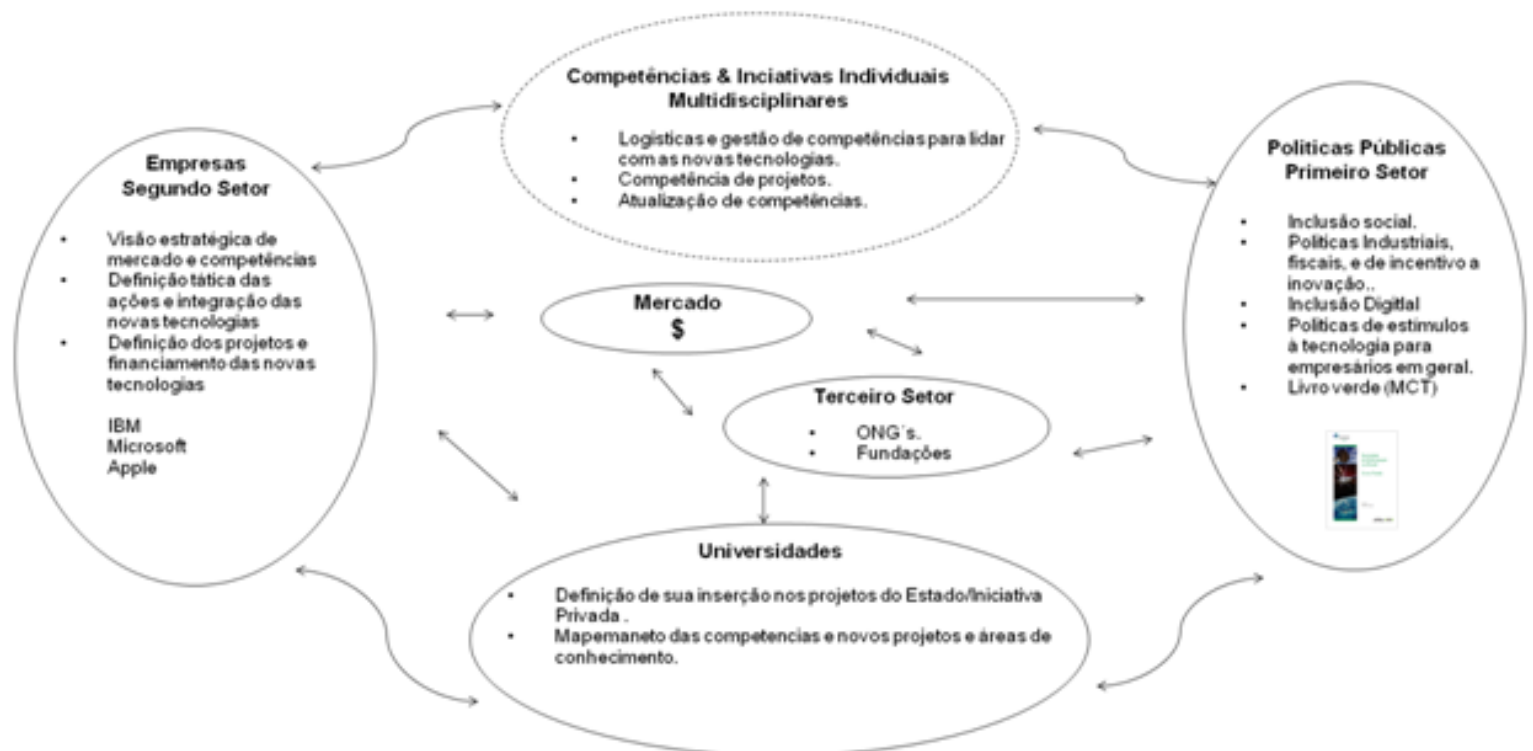

OZAKI m., POLIEZELLI L. Sociedade da Informação (organizadores). Ed. Saraiva São Paulo, 2008:7.

O esquema acima representa uma adaptação, em linhas gerais, do espaço das influências interrelacionadas que reverbera por todo ambiente sócio-tecnológico do ciberespaço sob a ótica do desenvolvimento de produtos e serviços distribuídos na rede, como também no ambiente restrito e controlado das corporações, políticas publicas e privadas relacionadas à utilização da tecnologia da 
informação. Numa dinâmica de atuação na qual os integrantes se relacionam numa necessidade cada vez maior, caracterizando a intensidade das relações do ambiente informacional e tecnológico, com características que influenciam os conceitos e praticas administrativas na gestão dos sistemas de informação representadas pelas arquitetura dos Serviços de negócios $\left(\mathrm{SOA}^{5}\right.$ e $\mathrm{BPM}^{6}$ ); em conjunto com as metodologias adaptativas para o desenvolvimento de sistemas (ALM, RAD, SCRUM, Fusion, RUP, etc.); e também dos aspectos sobre a Segurança da Informação no meio digital; e infraestrutura de comunicação/conectividade dos sistemas que utilizam a internet como plataforma de operação.

Por sua vez, diversos modelos organizacionais estratégicos, se apresentam formatados nos modelos e práticas orientados ao controle das informações: PMO - Gestão de Projetos; APMO - Agile PMO, estruturas de Governança Corporativa (ITIL, COBIT, SOX, SixSigma, etc.); em detrimento aos processos ágeis e flexíveis para atender as necessidades dinâmicas e disruptivas do mercado. Em relação aos aspectos da inovação dos sistemas informacionais, também é importante ressaltar as questões sociais que relacionam e transformam as necessidades de utilização da tecnologia pela sociedade. Que proporcionam, e até certo ponto orquestra o dinamismo das informações entre os usuários da rede em prol de um objetivo; que atrai, segmenta, e relaciona as informações no entorno dos atores que participam da Sociedade da Informação ${ }^{6}$. Ao passo que se torna cada vez mais evidente a questão inter-relacionada nas praticas de Gestão.

A Liderança de um gestor é um aspecto importante e diretamente relacionado à questão da Inovação com objetivo de mobilizar um grupo para exercer atividades relacionadas à mudança no aplicada na administração de um ambiente informacional. Perfil necessário ao entendimento e utilização das novas tecnologias que influenciam a sociedade contemporânea, agregado a fluência na comunicação, conhecimento e conceitos inerentes em todos os cenários da Sociedade da Informação ${ }^{7}$. Liderança que deve ser aplicada na influencia e processos para incentivar e colaborar ideias para o desenvolvimento de produtos. Podemos destacar alguns métodos que podem ser aplicados no comportamento das equipes para criação de um "mind set" que auxilia nas reflexões e busca de

\footnotetext{
5 SOA significa arquitetura voltada a serviço (Service Oriented Architecture) sob a tecnologia de Web Services que utilizam com os protocolos SOAP, UDDI e WSDL. Uma tecnologia sob ambiente Internet, disponibilizando os objetos de informação distribuídos.

${ }^{6}$ BPM/BPMS é a sigla de Business Process Management Systems que significa o gerênciamento dos processos de negócio. Solução que proporciona o desenvolvimento de sistemas para atender as novas expectativas dos níveis gerenciais e operacionais por meio da modularização dos serviços (SOA) na construção de sistemas, proporcionando maior colaboração (workflow) e alcance das informações distribuídas no meio digital.

${ }^{7}$ Sociedade da Informação : Conceito oriundo do Livro Verde elaborado pelo Ministério da Tecnologia (MCT) que aponta ações concretas, composta de planejamento, orçamento, execução e acompanhamento específicos do Programa Sociedade da Informação e Tecnologia. (http://www.mct.gov.br/index.php/content/view/18878.html)

4 Acronimos que representam metodologias de desenvolvimento rápido voltado para sistemas computacionais .

5 http://www.unifr.ch/econophysics/ , http://www.ge.infm.it/econophysics/
} 
soluções para inovação dos produtos e serviços intitulados: SIMPLEX, CPS-CREATIVE, Problem Solving, Método Classico, Seis Chapéus, Integral, dentre vários outros .

\section{CONCLUSÃO}

Uma das motivações desse artigo, no discorrer dos assuntos sobre inovação tecnológica e praticas de gestão orientada à inovação, está relacionada à carência da articulação dos conhecimentos necessários sobre as práticas de gerenciamento/gestão para prover um ambiente virtuoso necessário à dinâmica da mudança e criação de produtos inovadores. Um exemplo disso está representada numa matéria publicada no jornal da Folha de São Paulo sobre o assunto que apresenta a carência do mercado do perfil de gestores (Matéria sobre - Apagão de gestores lideres no mercado http://www1.folha.uol.com.br/fsp/empregos/ce2808201101.htm (acessado em 13-out-2012), representando um sintoma da carência de uma visão sistêmica e inter-relacionada cada vez mais necessária e crescente para gestão dos produtos e serviços a fim de acompanhar a dinâmica crescente das necessidades de negócio carregadas das alternativas e diversidade tecnológicas onde a Internet se apresenta como principal plataforma para o desenvolvimento de novas soluções ao mercado consumidor.

Outro diagnóstico está representado na participação das pequenas e medias empresas no mercado, em comparação a outros países, que representam e necessitam de maneira crescente de um ambiente inovador para se adaptarem à dinâmica do mercado. Na Itália, disciplinas e conceitos sobre de Administração/Gestão são introduzidas desde o ensino secundário com objetivo de incentivar a formação de empreendedores (PME's - Pequenas e Micro Empresas). Baseado nisso, podemos considerar como um resultado positivo a participação das PME's de 40\% no PIB. Acompanhado de outros países como na Alemanha onde as PME’s representam 60\% do PIB. Todavia, no Brasil, a taxa de falência ou extinção é de $75 \%$ nos primeiros anos e representam $20 \%$ do PIB brasileiro e responsável por $60 \%$ dos 94 milhões de empregos. Acompanhado da grande maioria das iniciativas vão à falência/fracasso no período de 3 a 5 anos segundo dados do SEBRAE e da FECOMERCIO. Sem deixar de considerar o viés positivo de crescimento desde 2000 quando a participação das PME's no setor produtivo obteve uma taxa de crescimento singelo de $4 \%$ e ganho de faturamento de $10,7 \%$ no período 2009-2010-2011. 


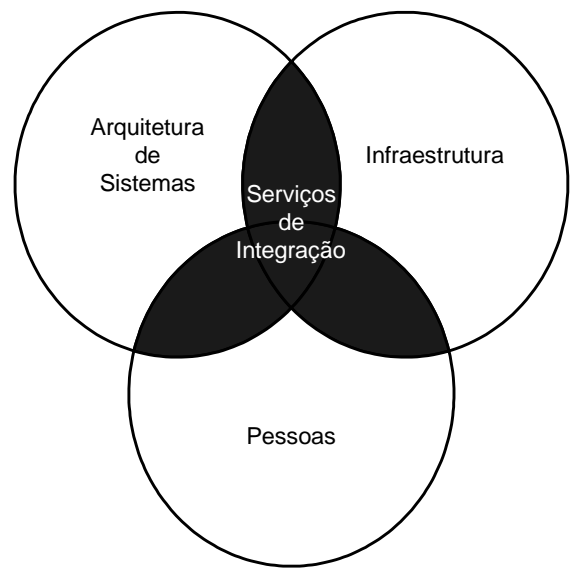

O contexto sobre as práticas de Gestão de um ambiente informacional, sob a ótica da Complexidade, representa uma perspectiva dinâmica e inter-relacionada que envolve um conjunto de fenômenos (sócio-politicos-administrativo-tecnológicos) no decorrer do tempo. Essa nova perspectiva representa uma nova configuração para colaboração das informações distribuídas no meio digital, agregando nas práticas de Gestão aos recursos tecnológicos da Internet que já se consolidou como principal ambiente de integração e distribuição das informações da sociedade contemporânea. O que é apresentado, de forma irreversível, a necessidade de uma nova perspectiva para interpretar os fenômenos do meio digital que se apresenta de forma cada vez mais interdisciplinar, coletiva e colaborativa integrada por meios meio dos serviços das soluções sistêmicas que relacionam as pessoas, tecnologia e a infraestrutura tecnológica.

A inovação não deve ser considerada como "modismo" devido a um eventual fortúnio financeiro ou marketing de balanço social ou propaganda da marca nos canais de comunicação para atingir um mercado consumidor. A inovação é operacional e deve se enquadrar e se caracterizar na cultura de investimento de uma organização. Sobretudo na percepção que associado a um produto considerado inovador, está associado um processo de inovação que não necessariamente está caracterizado por meio de um processo longitudinal e linear, mas em boa parte se apresenta de forma não linear, porém relacionados pelas diretrizes apresentadas nesse artigo. Como exemplo desse cenário, já assistimos com grande abrangência de utilização e sucesso, a consolidação da base de conhecimento SAFe (Scaled Agile Framework) com objetivo de proporcionar o desenvolvimento sustentável de produtos e serviços aderente ao inevitável processo de inovação dos produtos e serviços aderentes às necessidades de negócio do mercado em constante transformação.

A fim de evitar que os processos de inovação se tornem efêmeros quando devem se tornar parte integrante em toda a cadeia de valor e das práticas de gestão numa organização em prol do 
desenvolvimento dos produtos e serviços. É fundamental reiterar a proposta dessa abordagem como uma visão iterativa, constante, interdisciplinar e inter-relacionada numa dinâmica que inter-relaciona diversos movimentos tecnológicos de integração e desenvolvimento de sistemas, administração, processos, design, regras de negócio, cognição, ergonomia, integração homem-máquina, e interface de sistema. O que traduz numa aplicabilidade da Gestão na área de tecnologia numa relação primordial com demais áreas de conhecimento e setores de uma organização. 


\section{BIBLIOGRAFIA}

BROWN, John Seely, The Social Life of Information. Harvard Business School Press: Makron, 2001:129-223.

BURGELMAN, CHRISTENSEN, WHEEELWRIGHT. Strategic Management of Technology and Innovation - Quinta Edição, McGraw Hill, EUA, 2009:1-78:.

CAPRA Fritjof. As Conexões Ocultas, Editora Cultrix São Paulo, 2005:113-139.

CHAPPELL David. Enterprise Service Bus. O’REILLY, junho 2004:225-232.

ENGELBART Douglas. Augmenting Human Intellect s: A Conceptual Framework. October 1962:1119,70-79.

FINEGAN Andrew. Soft System Methodology : na alternative approach to knowledge elicitation in complex and poorly defined system - http://www.complexity.org.au/ci/vol01/finega01/html/ , acesso em 12/out/2012.

FREDERICK P., BROOKS Jr. No Silver Bullets - Essence and Accident in Software Engineering , University of North Carolina at Chapel Hill , January 1986: 6-16.

GARLAN David, SHAW Mary. An Introduction to Software Architecture, School of Computer Science Carnegie Melon, Pitsburg PA 15213-3890, January 1994: 5-21.

GETSCHKO, Demi. Observatório da Imprensa. São Paulo: TV Cultura, Quinta Feira 11 de março 2009 - ISSN 1519-7670 - Ano 13 n. 435.

GETSCHKO, Demi. Apresentação no programa Roda Viva https://www.youtube.com/watch?v=15sV-0cIPC8 . São Paulo: TV Cultura, Segunda Feira 13 de março 2009, acessado em 15/Nov/2012.

GETSCHKO, Demi: Apresentação Observatório da Imprensa: São Paulo, Quarta-Feira, 11 março 2009 - ISSN 1519-7670 - Ano 13 - No. 435 31/3/2009.

HASTIE, SHANE - InfoQ Governança -. http://www.infoq.com/news/2012/07/agile-ingovernment;jsessionid=D862273268EF1B60CB4304DCFBF4128B. Acesso em: 10 nov 2012.

LEFFINGWELL Dean : Agile Software Requirements , Boston MA, : Parson Education Inc., 2011:1116

MARIOTTI, Humberto: Pensando Diferente - Para lidar com a complexidade, Incerteza, e a Ilusão , Editora Atlas,São Paulo, 2010:113-174, 205-221.

MINTZBERG, Henry: The Power in and Around Organizations , Prentice Hall , London, 1983:291646.

MINTZBERG, Henry: The Raise and Fall Strategic Planning, The Free Press , EUA-NY, 1994:67158. 
NOBREGA clemente \& LIMA Adriano, Innovatrx - Inovação para não gênios, Editora Nova Fronteira , São Paulo, 2010:74-97.

SCHILLING, Melissa.Strategic Management of Technological Innovation - Third edition. NY University: McGraw-Hill, 2010:50-181.

PAES, Wander M. Interoperabilidade dos Dispositivos Móveis. São Paulo: PUC SP, 2008. 100 f. Dissertação de Mestrado - Programa de Pós-Graduação em Tecnologias da Inteligência e Design Digital, Faculdade de Tecnologia e Mídias, Pontifícia Universidade Católica de São Paulo, SP, 2008:30-33.

PAES, Wander M. Artigo Integracion \& Colaboracion em El Ciberspacio. Revista de Filosofia e Ciência, ISSN 1852-9488,

Año II | número 5 | septiembre-diciembre de 2011 | www.prometeica.com.ar, 2011:3-7, 8-13.

TAYLOR \& FRANCIS Group . Complexity Teory

http://cw.routledge.com/textbooks/9780415368780/a/ch1doc.asp, a, 2006, acessado em 15-out-2012. 